\title{
Dissecting Vertebral Artery Aneurysm Presenting Regrowth After Stent-Assisted Coil Embolization in Acute Stage
}

\author{
Takamichi Hijikata ${ }^{a}$, Eiichi Baba ${ }^{a}$, Kazutaka Shirokane ${ }^{a}$, Atsushi Tsuchiya ${ }^{b}$, \\ Motohiro Nomura ${ }^{\text {a, c }}$
}

\begin{abstract}
For a case of dissecting vertebral artery aneurysm (DVAA) in a dominant vertebral artery (VA) or posterior inferior cerebellar artery (PICA)-involving lesion, stent-assisted coil embolization (SACE) is an effective technique to preserve blood flow of the VA. A 41-year-old man presented with subarachnoid hemorrhage. Angiography demonstrated DVAA on the left VA just distal to the PICA, and the right VA was thinner than the left. For this case, SACE was performed to preserve the left VA and PICA. On the 10th day, angiography showed recurrence of the dissection. The dissected portion had thickened and extended to both distal and proximal sides involving the PICA origin and proximal portion to the PICA. A second endovascular embolization was performed and the recurrent dissecting aneurysm was embolized including the main VA cavity. In cases of DVAA, there is a possibility of recurrence after SACE, if a dissecting cavity remains unembolized. Therefore, total embolization is necessary under close observation from multiple angles, including the down-the-barrel view.
\end{abstract}

Keywords: Dissecting aneurysm; Vertebral artery; Recurrence; Stent-assisted; Embolization

\section{Introduction}

For dissecting vertebral artery aneurysms (DVAA), trapping by open surgery or internal trapping by an endovascular technique is a definitive treatment [1]. For a case of DVAA in a dominant vertebral artery (VA) or posterior inferior cerebellar artery (PICA)-involving lesion, stent-assisted coil embolization (SACE) is an effective technique to preserve the blood flow of the vertebrobasilar artery system. However, during SACE of DVAA, confirmation of the complete obliteration of a dissected cavity is difficult due to its anatomy and the exist-

Manuscript submitted March 4, 2018, accepted March 19, 2018

aDepartment of Neurosurgery, Kanto Rosai Hospital, Kawasaki, Japan ${ }^{b}$ Department of Neurology, Kanto Rosai Hospital, Kawasaki, Japan

${ }^{c}$ Corresponding Author: Motohiro Nomura, Department of Neurosurgery, Kanto Rosai Hospital, 1-1 Kizukisumiyoshi-cho, Nakahara-ku, Kawasaki 211-8510, Japan. Email: nomura413jp@yahoo.co.jp

doi: https://doi.org/10.14740/jocmr3397w ence of coils. There are some reports describing the recurrence of DVAA after SACE in the chronic stage $[2,3]$. However, there have been no reports describing the recurrence of DVAA in the acute stage. We encountered a patient with a ruptured DVAA treated with SACE, who showed aneurysmal regrowth 10 days after the initial treatment. In this report, we present our experience of the management of a ruptured DVAA, and discuss the radiological characteristics, etiology of recurrence, and pitfalls of treatment.

\section{Case Report}

A 41-year-old man without a significant medical history developed a headache while drinking alcohol at night. On the next day, he experienced the sudden onset of severe pain in the occipital region and neck, and vomited. He was brought to our hospital, and computed tomography (CT) revealed subarachnoid hemorrhage (SAH) (Fig. 1a). He showed right-arm convulsions and presented with transient respiratory arrest. After intratracheal intubation, angiography was performed. Angiography demonstrated DVAA on the left VA (Fig. 1b). The lesion was located just distal to the PICA, and the right VA diameter was smaller than that on the left (Fig. 1b). Therefore, for this case, SACE was performed to preserve the left VA and PICA. An 8-Fr guiding catheter (Optimo, Tokai Medical Products, Inc., Kasugai, Aichi, Japan) was advanced to the left VA directly originating from the aorta through a sheath placed in the right femoral artery. The working angles were LAO $67^{\circ}$ and CRA $7^{\circ}$ to visualize the VA and PICA origin, and AP and CRA $33^{\circ}$ to observe the inner cavity of the VA with a down-the-barrel view (Fig. 1c). A microcatheter for stenting (Plowler select plus, Johnson \& Johnson, Tokyo, Japan) was advanced distal to the dissecting portion. Another microcatheter (Excelcior SL-10, 45, Stryker, Tokyo, Japan) was advanced to the dissecting portion with a microguidewire (Transend EX, Softip, Boston Scientific Japan, Tokyo, Japan). Then, a few loops of a platinum coil were placed in the dissecting cavity. A stent (Enterprise VRD, $4.0 \times 23 \mathrm{~mm}$, Johnson \& Johnson) was advanced to the lesion through a microcatheter and deployed to cover the normal VA both distal and proximal to the lesion, including the dissected portion (Fig. 1c). Then, the dissected cavity was embolized with platinum coils. The lesion was embolized with a total of eight platinum coils $(43 \mathrm{~cm})$. Although an attempt to insert a ninth coil into the dissecting cavity was made, the microcatheter was expelled into the true lumen of the VA. Therefore, the procedure was com- 


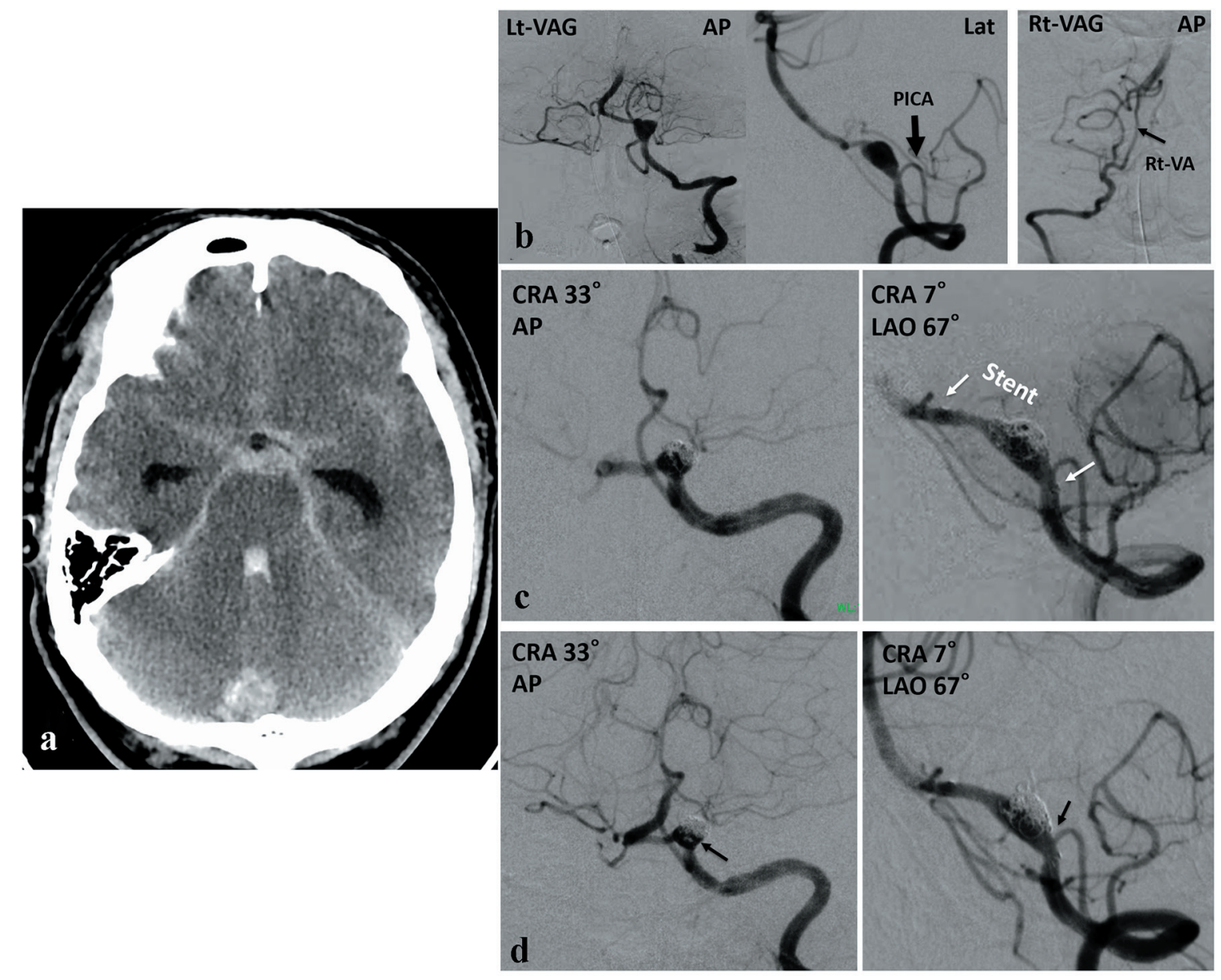

Figure 1. (a) CT demonstrating SAH. (b) Angiography demonstrating DVAA on the left VA. The PICA originating proximal to the dissected portion. The diameter of the affected left VA is thicker than that of the right. (c) Angiography showing two working angles. A stent is placed in the left VA, covering from distal normal VA to proximal to the PICA origin including dissection. (d) Postembolization angiography showing occlusion of the dissected portion and patency of the left VA. The residual cavity besides the VA and a small portion at the distal to the PICA origin are opacified (arrow).

pleted. After embolization, angiography with the down-the-barrel view revealed a residual cavity besides the main VA cavity, and a small residual cavity distal to the PICA origin (Fig. 1d). Post-operatively, he gained consciousness and showed no neurological deficit. From the third day, heparin was intravenously administered at 10,000 IU/24 h continuously.

On the 10th day, a routine angiography was performed to examine vasospasm. The angiography showed recurrence of the dissection (Fig. 2a). The dissected portion had thickened and enlarged to both distal and proximal sides. The dissected cavity involved the PICA origin, and it extended proximal to the PICA. The distal and proximal margins of the dissection were identical to the level of the stent tips. A second endovascular embolization was performed. The guiding catheter was advanced to the left VA as in the first treatment. Initially, microcatheter insertion was attempted to the recurred dissected cavity with the aid of a microguidewire through the stent cells. However, it was not possible. Although the contralateral VA was smaller than on the affected side, the diameter was not very small. Significant blood flow was expected. Therefore, it was planned to embolize the VA distal to the PICA.
The working angles were LAO $67^{\circ}$ and CRA $7^{\circ}$ to visualize the dissecting cavity, VA itself, and branching portion of the PICA. To preserve the PICA, a microcatheter (Excelsior SL$10,90^{\circ}$ ) was inserted to the left PICA with the aid of a microguidewire (Transend EX, Softip). A microcatheter (Excelsior 1018, Stryker) was advanced to the normal portion distal to the lesion. The recurrent dissecting aneurysm was embolized including the main cavity of the VA with a total of 10 platinum coils $(111 \mathrm{~cm})$ (Fig. 2b). After the second embolization, angiography demonstrated that the right VA supplied sufficient blood flow to the BA (Fig. 2c), and the left VA was occluded.

His post-operative course was uneventful. No symptoms due to vasospasm were observed. On the 45th day, ventricular peritoneal shunt was performed. Seventy-five days after the onset, he was transferred to another hospital for rehabilitation with disuse syndrome. The modified Rankin scale at discharge was 2 .

\section{Discussion}

For the treatment of DVAA, complete obliteration of the 

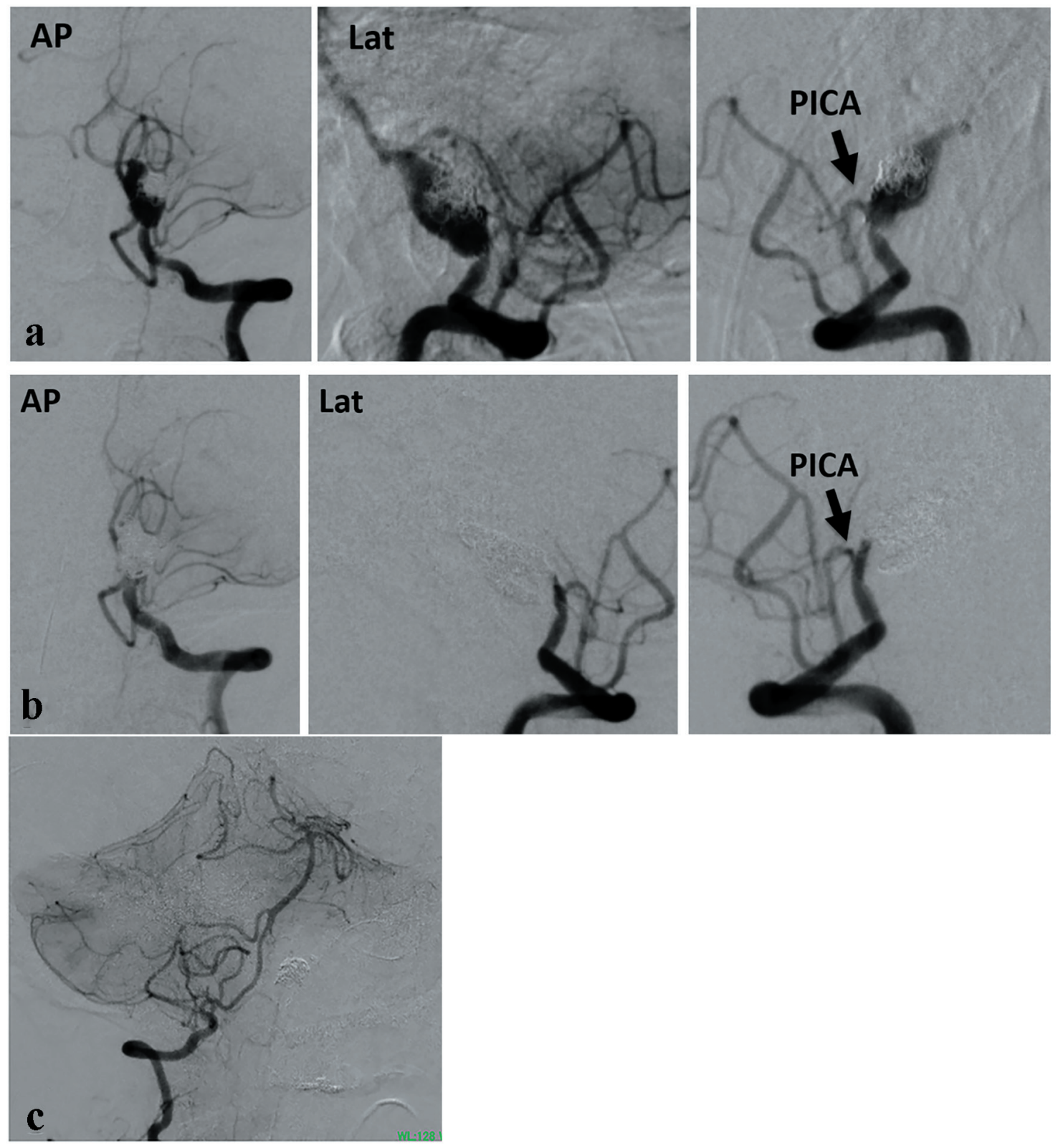

Figure 2. (a) Angiography performed on the 10th day showing recurrence of DVAA. Dissection was extended identical to the location of stent. (b) Post-embolization angiography showing complete occlusion of the left VA including aneurysm. A small part of the VA remains distal to the PICA. (c) The right VA has a significant diameter.

dissected cavity of the VA is optimal. However, in a case of DVAA with PICA involvement or on the dominant side, SACE is selected to preserve the patency of the VA or PICA. In such cases, there remains a possibility of recurrence. Regarding the recurrence of DVAA after SACE, major branch involvement and residual sac filling have been reported as predictors of recanalization [2]. In our case, a dissecting cavity partly remained after treatment, and the entry zone to the dissecting cavity was not clear. Due to the presence of the PICA, the proximal portion of the lesion was roughly embolized. Furthermore, besides the main cavity of the VA, the dissecting cavity remained. The blood flow into the residual dissected cavity might cause the enlargement of a dissected cavity hemodynamically in 10 days. The recurred dissection was restricted to the location of stenting. Blood flow entered via the entry point of dissection and spread both proximal and distal sides resulted in enlargement of dissection. At the both stent ends, dissection might not be extended due to compression by the stent tip.

As for the timing of recurrence or rupture of DVAA treated by SACE, Zhao et al [4] reported a case showing rebleeding $48 \mathrm{~h}$ after treatment. It was reported that rebleeding events occurred more frequently within 4 days after the initial treatment in cases of PICA-involving DVAA after single SACE or stent-only placement [1]. Jeon et al [2] reported 10 cases show- 
ing recanalization after 3 - 36 months (average: 18 months). Among them, additional treatment was performed for five cases. Song et al [3] reported six cases of DVAA presenting with recurrence 2 - 8 months after SACE. Shin et al [5] reported that one out of 14 DVAA cases treated with SACE showed recurrence. Although their case did not show rupture, recurrence was observed on angiography performed 1 year after the initial treatment. Internal trapping was performed, and a favorable outcome was achieved. Liu et al [6] reported five out of 72 patients with DVAA treated by SACE who showed recurrence. The timing of recurrence was between 3 and 8 months after the initial SACE. For these five cases, repeated SACE was performed in three, coiling of the aneurysm in one, and doublestent placement in one. Liu et al [6] analyzed the hemodynamics of the aneurysms, and concluded that the hemodynamics might play an important role in the recurrence of DVAA.

In a case of recurrence or rebleeding of DVAA, destructive treatment such as complete obliteration of the lesion including parent artery might be selected for immediate obliteration [4]. In such a case with a lesion involving the dominant VA, ischemic complications may occur. In our case, additional coil embolization with preservation of the main trunk of VA was initially attempted. However, it was not possible. Therefore, we performed embolization of the DVAA including the normal portion of the VA. For our case, the prevention of bleeding from the recurred aneurysm was considered more important than preservation of the VA patency, because the contralateral VA had a significant diameter, although it was thinner than the affected VA. Therefore, we selected internal trapping of the VA with preservation of the PICA. The PICA was successfully preserved, and no ischemic events occurred. However, for PICA-involving cases, additional stent placement in the lesion might be an alternative treatment [4]. Song et al [3] reported that treatment of a recurring DVAA, such as a second SACE of the aneurysm, double overlapping stent placement, and endovascular trapping, had been performed and was effective. For cases with PICA-involving DVAA, treatments with multiple stents and coils were reported to be preferable [1]. For a case with DVAA not suitable for simple internal trapping, SACE followed by a stent-within-a-stent technique has been reported to be effective [7]. For our case, we initially tried additional coil placement in the dissected cavity. However, it failed because the microcatheter could not be inserted through the stent cells. Therefore, we selected internal trapping to reduce risks of further growth and rerupture. However, additional stent placement may be an alternative.

\section{Conclusion}

In cases of DVAA, there is a possibility of recurrence after
SACE, if a dissecting cavity remains unembolized. Therefore, tight embolization is necessary under close observation from multiple angles including a down-the-barrel view.

\section{Acknowledgments}

This research was partly supported by research funds to promote hospital functions of the Japan Organization of Occupational Health and Safety.

\section{Conflict of Interest}

None.

\section{References}

1. Kim BM, Shin YS, Kim SH, Suh SH, Ihn YK, Kim DI, Kim DJ, et al. Incidence and risk factors of recurrence after endovascular treatment of intracranial vertebrobasilar dissecting aneurysms. Stroke. 2011;42(9):2425-2430.

2. Jeon JP, Cho YD, Rhim JK, Park JJ, Cho WS, Kang HS, Kim JE, et al. Stent-assisted coil embolization of vertebrobasilar dissecting aneurysms: procedural outcomes and factors for recanalization. Korean J Radiol. 2016;17(5):801-810.

3. Song Y, Wang Y, Li C, Wang Y, Mu S, Yang X. Retreatment and outcomes of recurrent intracranial vertebral artery dissecting aneurysms after stent assisted coiling: a single center experience. PLoS One. 2014;9(11):e113027.

4. Zhao KJ, Fang YB, Huang QH, Xu Y, Hong B, Li Q, Liu $\mathrm{JM}$, et al. Reconstructive treatment of ruptured intracranial spontaneous vertebral artery dissection aneurysms: long-term results and predictors of unfavorable outcomes. PLoS One. 2013;8(6):e67169.

5. Shin GW, Jeong HW. Endovascular treatment of intracranial vertebral artery dissecting aneurysms: follow up angiographic and clinical results of endovascular treatment in serial cases. Neurointervention. 2015;10(1):14-21.

6. Liu J, Jing L, Zhang Y, Song Y, Wang Y, Li C, Wang Y, et al. Successful retreatment of recurrent intracranial vertebral artery dissecting aneurysms after stent-assisted coil embolization: a self-controlled hemodynamic analysis. World Neurosurg. 2017;97:344-350.

7. Suh SH, Kim BM, Park SI, Kim DI, Shin YS, Kim EJ, Chung EC, et al. Stent-assisted coil embolization followed by a stent-within-a-stent technique for ruptured dissecting aneurysms of the intracranial vertebrobasilar artery. Clinical article. J Neurosurg. 2009;111(1):48-52. 